\title{
Supportive Care Program in CKD: from dream to reality
}

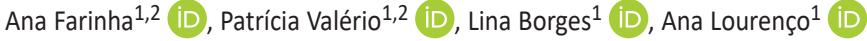 \\ ${ }^{1}$ Nephrology department, Centro Hospitalar de Setúbal, Setúbal, Portugal \\ 2 Núcleo de Estudos para o Tratamento Conservador da DRC, Sociedade Portuguesa de Nefrologia, Lisboa, Portugal
}

\begin{abstract}
"Deus quer, o homem sonha, a obra nasce"
(God wants, Man dreams, the Masterpiece is born)
\end{abstract}

Fernando Pessoa

\section{ABSTRACT}

The number of older and frail patients has greatly increased in nephrology departments. Traditional treatment options have become inappropriate because they seem unable to achieve goals such as quality of life. Principles from palliative care have been brought to chronic kidney disease management with good results; thus a new way to approach these patients has been reported. In Portugal, in 2011, Direcção Geral de Saúde published a guideline to substantiate supportive care as an option, for certain patients, in certain circumstances. The best evidence known at the time was stated and the conditions that should be met were regulated, but limited information was given about practical implementation of what. In this article we describe our experience with a supportive care program from planning to the implementation steps, since 2015. We reflect on the flowchart, the barriers, the conquests, and the data up to the end of 2020.

Keywords: Chronic Kidney Disease, Implementation, Palliative Care, Supportive Care Program

(C) 2021 Portuguese Journal of Nephrology \& Hypertension. Published by Publicações Ciência \& Vida This is an open access article under the CC BY-NC-ND license (http://creativecommons.org/licenses/by-nc-nd/4.0/).

\section{INTRODUCTION}

All nephrologists have at some point felt uncomfortable about the way some patients live in dialysis. That feeling of not doing enough was our impetus to look for options. We started studying solutions for patients who did not do so well on dialysis and the literature gave us the answer: a palliative approach should support patients who choose not to undergo any kind of kidney replacement therapy (KRT) or who are unable to proceed to it, in parallel to what happens in oncology when patients are not suitable for curative procedures.

The new vision of palliative care (PC) is, indeed, a perfect definition to fit in our chronic kidney disease (CKD) patients: an answer to the needs of patients with advanced, incurable and progressive disease, and / or with intense suffering, with multiple symptoms evolving, with the main objective of ensuring the best quality of life for the patient and family ${ }^{1}$. That is also the answer to the problem of not abandoning these patients when dialysis is not the way. In cases where KRT fails to improve survival, quality of life, or functional status ${ }^{2}, P C$ has emerged as an ethical and organizational imperative - a human right.

In the last decade, growing evidence has proven that, in older and frail patients, supportive care not only provides time to live but also wellbeing ${ }^{3}$. Based on these studies, in Portugal, in 2011, Direç̧ão Geral de Saúde (DGS) published a guideline to validate supportive care as an option. It regulated that "all therapeutic measures should be applied without dialysis or transplantation, when kidney replacement therapy (KRT) is not indicated or not possible or when it does not provide a superior quality of life to that offered by supportive care ${ }^{\prime \prime}$, but limited information was given about practical implementation. This article shares our experience in the planning and implementation of our supportive care program since 2015. We reflect on the flowchart, the barriers we must overcome, the conquests, and the data up to the end of 2020 .

\section{PRINCIPLES AND GOALS}

As stated in the DGS guideline, all our CKD stage 4 and 5 patients have an appointment where they are enlightened about options for coping with their kidney failure. As demanded by DGS, KRT and supportive care are presented, and details about what to expect in each modality are given. If we were going to give patients the option not to choose dialysis, we would have to build a structured program to support them. So, we created an individualized program, with a dedicated team to guarantee some key elements. The team would have to:

1 - Have advanced knowledge of palliative care but also to be familiar with CKD stage 5 management and specificities;

2 - Recognize prognosis (both from CKD and vital status); 
3 - Be aware of approaches to control symptoms, to provide better quality of life (QoL) or improve functional status, as well as how to keep residual kidney function and control kidney failure complications (anemia, CKD-MBD, hyperkalemia, metabolic acidosis, fluid overload, and so on);

3 - Afford total accessibility to patients and families, promoting the feeling of accompaniment and care, promoting empathy and knowledge of each family's characteristics;

4 - Perform an intervention centered on the person and not on the disease;

5 - Have good enough communication skills to conduct advance care planning and break bad news;

6 - Prepare patient and family for end of life (EoL), explore all possible scenarios and available solutions and to support bereavement;

5 - Work together with differentiated palliative care teams (community or in-hospital) in complex cases.

\section{IMPLEMENTATION}

Although ambitious in goals, to implement this option is not as difficult as it might seem. The program development started at the beginning of 2014 and took about 6 months. We present the plan we used.

\section{First step: education}

The supportive care approach needs differentiation as any other modality of kidney replacement therapy. In Portugal, there is no basic

\section{Table 1}

Basic Curriculum of a Supportive Care Program

Optimized primary supportive care

Multidisciplinary renal teams

Competences:

(a) Identify those patients who are most likely to benefit from supportive care interventions.

(b) Assess and manage symptoms effectively.

(c) Estimate and communicate prognosis (survival and future illness trajectory) to the best of their ability.

(d) Develop appropriate goals of care that address individual patients' preferences, goals, and values.

(e) Possess knowledge of, and experience with, available local supportive care services, and be aware of when and how to refer.

(f) Assist with care coordination including referral to specialist supportive care and hospice service as available and appropriate

Adapted from Davison SN, Levin A, Moss AH, Jha V, Brown EA, Brenann F, et al. Executive Summary of the KDIGO Controversies Conference on Supportive Care in Chronic Kidney Disease: developing a roadmap to improving quality care. Kidney Int 2015; 88(3): 447-59.

curriculum designed to guide this education, but some articles from countries where supportive care is already established gave us some crucial clues. Kidney Disease: Improving Global Outcomes (KDIGO), in partnership with the International Society of Nephrology, has also defined basic knowledge ${ }^{5}$ (Table 1 ).

\section{Second step: the team}

We organized a multidisciplinary group (the only way to fully respond to the different needs of each patient and family) composed of nephrologist, nurse, social worker, psychologist, and nutritionist. The first three people have training in palliative care. The consultation is made with both doctor and nurse. The other members of the team are consulted whenever needed. From the very first beginning, the in-hospital palliative team was involved in the discussion of difficult cases or the referral of complex ones. We also work with the community palliative team and with primary care facilities.

\section{Third step: resources}

There is no set time arranged for the consultation. We perform it once a week as part of a planned schedule or at any time as required on an urgent basis. It is done in a room in the hemodialysis unit. The room has space for the team ( 2 members), the patient (even if on a stretcher or in a wheelchair) and the family. For equipment, in addition to the computer used for registration, we have a sphygmomanometer and a weighing scale. We can also access palliative care medication (e.g. fentanyl) or diverse material for interventions (wound dressing, urinary catheterization, ultrasound and so on) if necessary.

But the main resource needed is TIME! All nephrologists feel the pressure of having a huge number of patients and little time to care for them.

\section{Forth step: referral}

After the appointment where modalities of kidney replacement therapies are presented to patients and families, when the patient opts for supportive care, he is referred to the supportive care program. All suitable modalities are presented to each patient. Whenever the patient could choose, his option is respected. In the first supportive care appointment, motivations and goals of care are explored. Depression, cognitive impairment, or any myths that might influence this choice are ruled out. If the patient is sure about his option, the follow-up will proceed. We also follow patients who have significant comorbidities that determine a bad prognosis, and in whom we believe KRT will not alter prognosis. This decision is made by the nephrologists of the department and shared with the family (whenever the patient does not possess the ability to decide). Some clues that suggest worse life expectancy were used to guide nephrologists and highlight patient selection:

1. Answer no to the surprise question (would you be surprised if this patient were to die in the next 6 months/one year?);

2. Age $>80$ years old;

3. Institutionalized patient;

4. Serum albumin $<3 \mathrm{~g} / \mathrm{dl}$;

5. Dementia.

Glomerular filtration rate is not used to establish a cut-off for referral. In the same way, we generally don't use scores published elsewhere (e.g. Bansal score for pre-dialysis patients or Cohen or REIN score for dialysis patients) because they are not validated for our Portuguese 
population, and they may not reflect the reality of an individual patient. But we assess frailty, mostly by the Canadian Frailty Score ${ }^{6}$ and the Edmonton Frailty Scale ${ }^{7}$.

The main principle was to follow the patients who might benefit the most from our dedicated team.

\section{THE MODEL IN PRACTICE}

\section{Workup}

A first support care program consultation takes at least one hour. That is the time needed to explore:

1. Patient and family's social background, context and specific needs;

2. Patient motivation to choose supportive care, to highlight myths or misunderstandings; to exclude depression or other reversible factors that might condition this choice;

3. Adequate emotional support and communication between the patient, family and therapeutic team, in order to establish an empathetic, open and trusting relationship;

4. Family support: they live and share the patient's suffering so they need greater attention and availability. The goal is to clarify, inform, demystify or develop strategies to deal with / solve problems that may arise;

5. Clinical context, comorbidities, medication, functional limitations and rehabilitation potential;

6. Symptom control: recognize, assess, and adequately treat the multiple symptoms that arise and have direct repercussions on the patient's well-being. The changes occur very quickly, which implies constant monitoring of symptoms and a frequent therapeutic reassessment.

\section{Table 2}

Supportive Care template

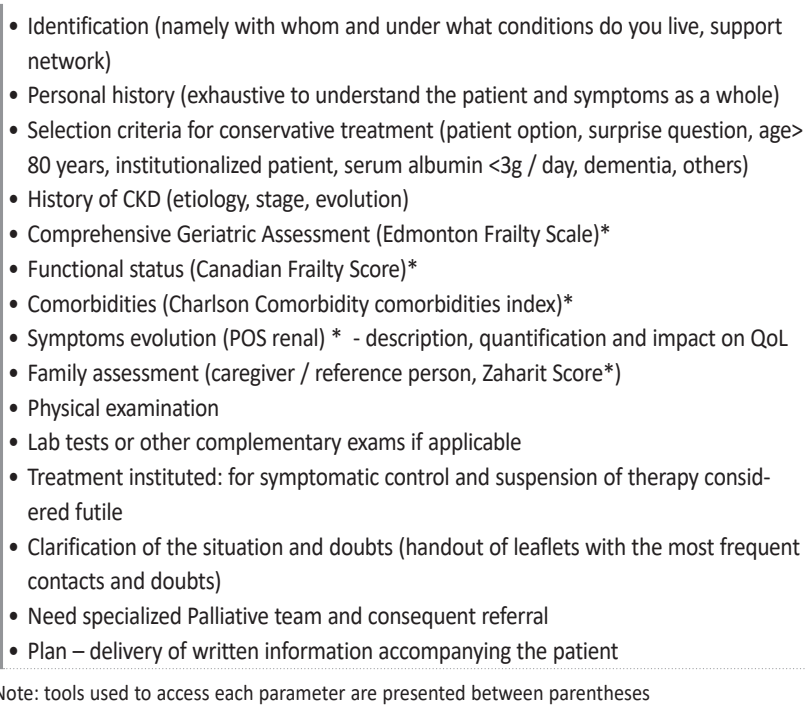

We used a template to standardize information and unify concepts and approaches in the team. The goal is to make everyone comfortable and useful in his role to optimize our interventions (Table 2).

Special attention is paid to symptom control. The nurse applies POSRenal ${ }^{8}$ and the doctor institutes a treatment plan. Therapeutic review and reconciliation are done in order to avoid futile medication and not to forgo therapy that promotes comfort, quality of life or prognosis modification. At the end of the appointment, we summarize all decisions made and provide written information on comorbidities, prognosis, symptoms, planning and contacts to both primary care physician and to family/caregiver (information is adapted to its target reader).

Follow-up visits are scheduled depending on the severity of the situation and need to access interventions. Some contacts are made by phone. The mean follow-up time is every 2-3 months.

\section{Hospitalization and hospice referral}

We are aware that our experience is limited (even if it has been increasing and we became more confident with these patients), so some of the times, discussion or even referral for hospital PC team or for hospice is needed (see data below).

Also, even with our bigger effort to promote care at patients' home, in some situations hospitalization is needed.

The main reasons for hospitalization or hospice referral are:

1. Lack of caregiver: patient is unable to stay at home because of no caregiver, caregiver unable to assume support, caregiver exhaustion or family conflict;

2. Complex symptoms, difficult to solve at home;

3. Unexpected worsening condition;

4. Unavailable place in the palliative care network (mainly in the community team) to cope with complex cases.

\section{BARRIERS}

The most important barrier to implementation of any project, in any area, is unfamiliarity. We faced distrust by some colleagues, a few of them not even nephrologists, but from other specialties that also follow the patients for other issues and push them to accept dialysis.

Patient health illiteracy or cognitive impairment has also been an important obstacle. It may be difficult to understand if patients have really internalized all the implications of the available options, especially when this requires time-consuming conversations with untrained or inexperienced stakeholders. In our experience, we faced no litigation or conflict; we never felt that expectations (from the patient or family/ caregiver) were beyond what we could provide.

At the institutional level, we still face a lot of hurdles, common to all institutions, such as lack of economic aid, reimbursement policy, activity regulation, home-based care, and community support. We are still struggling to overcome these obstacles (Table 3). 


\section{Table 3}

Barriers to implementation of supportive care program in CKD

\begin{tabular}{l|l||l}
\multicolumn{1}{c|}{ Related to patients and families } & \multicolumn{1}{c|}{ Related to Institutions / doctors } & \multicolumn{1}{c}{ Related to central administration } \\
\hline - Lack of realistic information (prognosis, QoL) & - Lack of education & $\bullet$ Lack of reimbursement policy \\
- Health illiteracy & - Religious beliefs & Lack of same rights as others RRT: \\
- Religious beliefs & - Fear of death /defeat & - Transports \\
- Fear of abandon & - Lack of human or logistic resources & - Taxes \\
- Lack of caregiver & - Lack of support in community (e.g. home visiting) & - Free medication \\
- Very frail /dependent patients & - Lack of 24-hour availability (e.g. phone prevention) & - Lack of data (e.g. national registry) \\
- Severe comorbidities & & - Lack of cost-benefit evaluation \\
\hline$>$ Need to institutionalization / hospital inpatient & &
\end{tabular}

\section{PILLARS FOR GROWTH}

From the conceptual framework to the practical, we defined four main pillars to grow our program and wisdom:

1) Identification of patients and its palliative needs - improve education and discrimination capacity for prognostication, earlier involvement and better care provision for both supportive care team and other nephrologists that we work with;

2) Advance care planning and patient and family support - to improve communication skills and training both for supportive care team and colleagues, especially residents;

3) Symptom assessment \& management - to have a home visit, performed by the team nurse to better support patient and family, to identify local needs;

4) Caring for the dying patient \& bereavement - to have an established appointment to support families who lose their loved ones.

To promote the first pillar, we hold an educational clinical session and promote patient discussion whenever it seems reasonable. We also built a program for residents education in our unit.

The other pillars are team based and patient centered. At a team level, we continued our education and training. We promote team strength by sharing experiences on each case and anticipate the plan. To implement the last two pillars, we still fight for more time and resources.

\section{RESULTS}

From July 2015 until December 2020, we followed 88 patients: 26 who were on renal replacement therapy (RRT) (group 1) and meant to withdraw and 62 who were referred because of the option not to perform any RRT (group 2). Their main characteristics are presented in Table 4. In Group 1, 11 patients maintained palliative hemodialysis and 15 withdrew completely. The main cause for withholding RRT was malignancy in advanced stage, followed by dementia and frailty.

In group 2, 33 patients chose freely not to perform RRT. In the remaining cases, the decision was made by the assistant nephrologist and family because of patient's lack of capacity to decide. In all but 3 patients, the surprise question was positive. The main causes for denying RRT were dementia $(19,30.6 \%)$, frailty $(13,21 \%)$, multiple comorbidities (12, $19.4 \%)$. Seven patients had no other cause than their informed option.

In group 1, the main symptoms that we had to deal with were mostly related to the main-comorbidity that conditioned prognosis (mostly cancer). In group 2, neuropsychological symptoms were the core concern (fatigue, lack of mobility, cognitive impairment, loneliness). Pain, dyspnea, or nausea were not frequent and were easily controlled.

The mean number of patients in follow up at same time is about 13.

We also follow inpatients, who are hospitalized in our nephrology department, as shown in Graphic 1. The main reason for these patients

\section{Table 4}

Main characteristics of the two groups followed in supportive care program

\begin{tabular}{l|l||l}
\multicolumn{1}{c|}{ Group 1 - Withdraw RRT } & Group 2 - Option \\
\hline Number & 26 & 62 \\
\hline Age, years (mean, min-max) & $72.9(44-91)$ & $84.6(67-97)$ \\
Gender, female (n, \%) & $13(50 \%)$ & $34(54.8 \%)$ \\
Origin & Inpatient/private facility & Nephrology outpatient consultation \\
Institutionalization (n, \%) & $6(27 \%)$ & $37(59.7 \%)$ \\
\hline Karnofski scale (mean) & 35 & 42 \\
\hline Charlson Comorbidity Index (mean) & 11.2 & 10 \\
Hospice referral (n, \%) & $4(15.4 \%)$ & $4(6.5 \%)$ \\
\hline Follow-up time, days (n, \%) & $17(2-66)$ & $136(6-1047)$
\end{tabular}




\section{Graphic 1}

Evolution on the number of patients treated over the time by our supportive care program

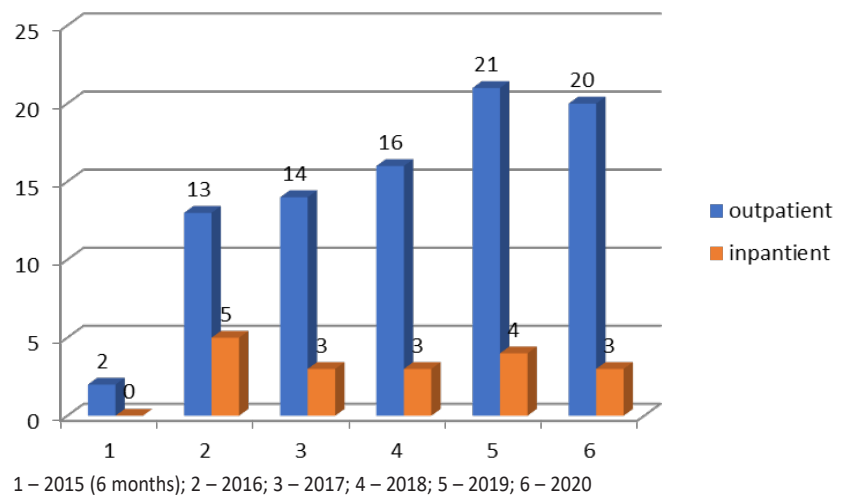

being admitted was a new comorbidity (cardiovascular event, cancer diagnosis) or decline in functional status /cachexia. In these situations, a team evaluation is performed when dialysis is no longer a suitable option, family is included in the prognosis discussion, and a plan is drawn up to provide comfort measures.

\section{CONQUESTS}

Some colleagues feared that referral to our appointment would mean denying treatment or depriving hope. The growing number of patients (Graphic 1) from the implementation to now reflects the confidence in our work but also the evidence in the capital gains that this option adds to CKD care.

We also felt that our program meets patients and families' goals of care.

We know that it has not been perfect all the time; we know that it is not what we expect every day, but we truly believe that offering something is maybe better than offering nothing. We also have faith that the first step is the hardest, but also the most important.

\section{THE FUTURE}

We have planned for the future:

1) Patient care: to expand our time for consultation, dedicating more time to the earlier phases of the disease, to perform home visitation by our nurse, to improve our geriatric care in rehabilitation (with physiotherapist, occupational therapist);

2) Education: to disseminate our experience and our knowledge so we all speak the same language at the professional level but also at the patient level;

3) Audit: to achieve quality criteria given by international guidelines for key quality indicators.

The COVID19 pandemic limited our time of consultation, our human and logistic resources, and our community support. But even so, we did not drop this activity.

\section{CONCLUSION}

Whenever conditions vary or evolve, we have to adapt our practice to progress and provide the best care for our patients. Changing is always difficult and challenging, but one small step for a department may represent a big step for a community. Our program was a novelty at the time of conception, but it has been raising awareness of palliative care for CKD patients. We prioritized quality of life in a patientcentered approach. The beginning may always be ruled by inertia and resistance, by fears such as lack of reliable prognostication or of giving hope way but we should focus on overcoming these barriers. To share our experiences might create evidence and the confidence to achieve these goals. It is also important to build a common language to reproduce and compare programs so we can implement a constant improvement in the services provided. We consider our program simple to apply so we hope it might help other departments to start their own plans to provide the best care for our patients.

\section{Acknowledges}

To Prof. Josefina Santos: for the review and counseling.

To Dr. José Vinhas: because he believed in the project; he believed in our team and supported it to make it possible.

Disclosure of potential conflicts of interest: none declared.

Presented data were approved by Hospital Ethics Committee.

\section{References}

1. WHO Definition of Palliative Care. World Health Organization. http://www.who.int/cancer/palliative/definition/en/ (Accessed 30 Sep 2021)

2. Farinha A. Prognostication in end-stage renal disease. Port J Nephrol Hypert. 2016; 30(4): 246-251.

3. Buur LE, Madsen JE, Eidemak I, Krarup E, Lauridsen TG, Taasti LH, Finderup J. Does conservative kidney management offer a quantity or quality of life benefit compared to dialysis? A systematic review. BMC Nephrology. 2021;22:307.

4. https://www.dgs.pt/directrizes-da-dgs/normas-e-circulares-normativas/ norma-n-0172011-de-28092011-atualizada-a-14062012-jpg.aspx. Accessed 21th sep 2021

5. Davison SN, Levin A, Moss AH, Jha V, Brown EA, Brennan F et al. Executive summary of the KDIGO Controversies Conference on Supportive Care in Chronic Kidney Disease: Developing a roadmap to improving quality care. Kidney Int. 2015;88:447-459.

6. Foster R, Walker S, Brar R, Hiebert B, Komenda P, Rigatto C et al. Cognitive Impairment in Advanced Chronic Kidney Disease: The Canadian Frailty Observation and Interventions Trial. Am J Nephrol. 2016;44(6):473-480.

7. Davison SN, Jassal SV. Supportive care: integration of patient-centered kidney care to manage symptoms and geriatric syndromes. Clin J Am Soc Nephrol. 2016;11(10):1882-1891.

8. Raj R, Ahuja K, Frandsen M, Murtagh FEM, Jose MJ. Validation of the IPOS- Renal Symptom Survey in Advanced Kidney Disease: A Cross-sectional Study. Pain Symptom Manage. 2018;56(2):281-287.

\section{ORCID}

Ana Farinha (iD) 0000-0002-5402-7626

Patrícia Valério (iD) 0000-0001-7397-0233

Lina Borges (iD) 0000-0002-1176-7720

Ana Lourenço (iD 0000-0002-6896-9665

\section{Correspondence to:}

Ana Farinha, MD

E-mail: alpfarinha@gmail.com 